\title{
AFM detection of biophysical characteristics of specific regulatory $T$ Cells after allogeneic hematopoietic stem cell transplantation
}

\author{
Xu Wang ${ }^{1 \dagger}$, Zhenyi Jin ${ }^{2+}$, Xinyu Wang ${ }^{2}$, Shaohua Chen ${ }^{2}$, Lijian Yang ${ }^{2}$, Kanger Zhu ${ }^{3}$, Xiuli Wu $\mathrm{Wu}^{1,2^{*}}$ and Yangqiu Li $\mathrm{Li}^{1,2^{*}}$
}

\begin{abstract}
Background: Based on the dynamics of antigen recognition from T cell receptor complex and T cell activation, different function of T cells may present different morphology feature, at least at molecular level. Regulatory $T$ (Treg) cells play a critic role in regulation the onset of graft versus host disease (GVHD) after allogeneic hematopoietic stem cell transplantation (allo-HSCT). Little is known about their morphology feature which may relate to the regulating function in GVHD.

Results: In this study, we detected the biophysical architectural changes of specific Treg cells subsets after allo-HSCT using biological atomic force microscopy to characterize their biological characteristics and improve our understanding of cell structure-function relationships in GVHD. There were dramatic overall shape and surface membrane deformations of the Treg cells associated with patients at GVHD onset or without GVHD. The Treg cells at GVHD onset or without GVHD could be distinguished by the morphologic parameters of morphology, membrane nanostructure, and membrane pore. These AFM parameters of Treg cells morphology differed obviously between GVHD and without GVHD. The multiple irregular microspikes could be observed on the surface of Treg cells without GVHD.
\end{abstract}

Conclusions: The remarkable biological morphology changes in the cell membrane structure of Treg cells after allo-HSCT might be related to the function of Treg cells which could inhibit the occurrence and development of GVHD.

Keywords: Atomic force microscopy, Regulatory T cells, Graft versus host disease, Allogeneic hematopoietic stem cell transplantation

\section{Background}

Allogeneic hematopoietic stem cell transplantation (alloHSCT) is an important therapeutic option for a number of malignant and refractory hematological diseases. Graft versus host disease (GVHD) is the main complications following allo-HSCT. GVHD is primarily a donor T cellmediated syndrome whereby $\mathrm{T}$ cells in the graft elicit an immune response, resulting in host tissue damage [1,2]. Knowledge of the immunobiology underlying GVHD has advanced by virtue of immunology research in animal models, as well as clinical observations. GVHD occurs as a

\footnotetext{
*Correspondence: siulier@163.com; yangqiuli@hotmail.com

${ }^{\dagger}$ Equal contributors

${ }^{1}$ Key Laboratory for Regenerative Medicine of Ministry of Education, Jinan University, Guangzhou 510632, China

${ }^{2}$ Institute of Hematology, Jinan University, Guangzhou 510632, China

Full list of author information is available at the end of the article
}

result of $\mathrm{T}$ cell activation followed by allo-reactive $\mathrm{T}$ cell expansion and differentiation [3]. Since GVHD is characterized by the loss of tolerance and the development of autoimmune manifestations, it is reasonable to indicate that a deficiency in regulatory $\mathrm{T}$ (Treg) cells reconstitution plays a critical role in GVHD pathophysiology and might participate in mediating GVHD and graft-versus-leukemia (GVL) effect after allo-HSCT $[4,5]$. The Treg cells, which identified suppressive population of $\mathrm{CD} 4+\mathrm{T}$ cells that expressed high levels of the IL-2 receptor $\alpha$-chain (CD25) formerly known as suppressor $\mathrm{T}$ cells, are a subpopulation of $\mathrm{T}$ cells which modulate the immune system, maintain tolerance to self-antigens, and abrogate autoimmune disease $[1,6,7]$. Mouse models have suggested that modulation of Treg cells can treat autoimmune disease and cancer, and facilitate organ transplantation $[1,3,8]$, while the molecular mechanism of Treg cells is still unclear. Rapid 
developments in biological atomic force microscope (AFM) have allowed researchers to investigate the behavior of single living cells $[9,10]$. Using AFM to understand the correlation between intracellular chemical processes and cellular physical properties will significantly improve our understanding of physiological events. All the changes in cell ultrastructure help us to better understand the structure-function relationships. The AFM applications have the possibility to detect the early subtle changes before significant pathology occur and to give the decision which time to terminate the treatment $[11,12]$. Thus, to further understand the biological characteristics of specific Treg cells subsets related to GVHD, in this study, we detected the biophysical architectural changes of specific Treg cells subsets after allo-HSCT using biological AFM.

\section{Methods}

\section{Samples}

Thirty leukemia patients (15 males and 15 females, median age, 30.6 years; range, $20 \sim 46$ years) were monitored prospectively after allo-HSCT. The diagnosis was based on the criteria reported in the guidelines of American Society of Hematology. According to WHO classification [13], those 30 patients with leukemia included 16 cases with acute myeloid leukemia (AML), 10 cases with chronic myeloid leukemia (CML), and 4 cases with acute lymphoid leukemia (ALL). Acute and chronic GVHD were diagnosed and graded as previously described [14].

\section{Ethics and consent statement}

All of the procedures were conducted according to the guidelines of the Medical Ethics Committee of the health bureau of the Guangdong Province of China (Registration number: NUMD-201303), in compliance with the Helsinki Declaration. Likewise, adult participants in the study (all human subjects) provided informed consent for blood donation.

\section{Treg cells isolation}

The Treg cells were obtained from freshly acquired peripheral blood samples in patients at GVHD onset and patients who did not develop GVHD at the same time point. Peripheral blood mononuclear cells (PBMCs) were isolated from peripheral blood samples by Ficoll-Hypaque gradient centrifugation. The CD4 + CD25+ Treg cells were sorted from PBMCs using human CD4 + CD25+ Treg cells isolation Kit and $\mathrm{MACS}^{\bullet}$ magnetic cell sorting technique (Miltenyi Biotec, Bergisch Gladbach, Germany) [15].

\section{Atomic force microscope measurement}

The cell suspension (CD4 + CD25+ cells from cases with GVHD, CD4 + CD25- cells from cases with GVHD, CD4 + CD25+ cells from cases without GVHD, CD4 +
CD25- cells from cases without GVHD respectively) was dropped on glass cover slips and fixed in $4 \%$ paraformaldehyde and air dried prior to atomic force microscope (AFM) scanning and phasecontrast micrograph. Topographic images of cells were obtained using an AFM (Autoprobe CP Research, Veeco) in contact mode. In all AFM experiments, gold-coated silicon nitride tips (UL20B; Park Scientific Instruments) with a spring constant of $2.5 \mathrm{~N} / \mathrm{m}$ and a tip diameter of $20 \mathrm{~nm}$ were used. An optical microscope was used to help select the desired cells and direct the position of the AFM tip. Single-cell imaging was performed for five cells of each condition, and each cell was scanned three times. To gain information on the topography of the cell, all images were analyzed using the instrument-equipped IP2.1 software (Veeco Metrology Group, Santa Barbara, CA). The area analyzed with AFM was a $10 \mu \mathrm{m} \times 10 \mu \mathrm{m}$ square. The length (L) and width (W) defined the maximum and minimum values of the cell diameter, respectively. The $\mathrm{r}$ defines the ratio of $\mathrm{L} / \mathrm{W}$. The peak $(\mathrm{H})$ value means the maximum height values of the cell membrane surface. The surface roughness value $\mathrm{Ra}$ was analyzed for each sample. This value defined the mean value of the surface roughness in the area being analyzed. All these parameters were directly determined using the IP2.1 software $[12,16]$.

Roughness: $\mathrm{Ra}=(1 / \mathrm{N}) \sum_{n=1}^{N}\left|Z_{n}-\bar{Z}\right| . \bar{Z}$ : given by the average height within the included areas defined by a region group $\bar{Z}=(1 / \mathrm{N}) \sum_{n=1}^{N} \mathrm{Z}_{\mathrm{n}}$. The $Z$ is the height profile line based on the surface.

\section{Statistical analysis}

Differences between numerical variables were calculated by means of the one-way ANOVA (the Welch test). The $P$ values were two tailed, and $P<0.05$ was considered statistically significant. The SPSS software package 13.0 (SPSS, Chicago, IL) was used for all data analysis.

\section{Results and discussion}

All patients achieved hematopoietic reconstitution. The median time for neutrophils (absolute neutrophils counts $>0.5 \times 10^{9} / \mathrm{L}$ ) and platelets (platelets counts $>$ $20 \times 10^{9} / \mathrm{L}$ ) engraftment was 12 and 15 days, respectively. Of the 30 patients, the incidence of total GVHD was $73.3 \%(n=22)$, while 8 patients were without acute or chronic GVHD. The incidence of $\mathrm{I}^{\circ} \sim \mathrm{IV}^{\circ}$ and $\mathrm{III}^{\circ} \sim \mathrm{IV}^{\circ}$ acute GVHD including acute GVHD after transplantation was $63.3 \%(n=19)$ and $13.3 \%(n=4)$, respectively. Of the 30 patients who survived more than 100 days after transplantation, the incidence of limited and extensive chronic GVHD was $33.3 \%(n=10)$ and $6.7 \%(n=2)$, respectively. Therefore, in this study, we compared the biophysical 
properties of Treg cells from patients without GVHD (8 cases) and patients with GVHD (including acute and/or chronic GVHD, 22 cases) after allo-HSCT.

The AFM was used to study the biophysical properties (the parameters including morphology, membrane nanostructure, membrane pore, adhesion, distribution and so on) of Treg cells at GVHD onset or without GVHD. The overall shape of Treg cell was shown an oval shape. The $r(\mathrm{~L} / \mathrm{W})$ value was nearly equal to 1 . The AFM morphological images of Treg cells were shown in Figure 1. The total CD4 + CD25+ cells (including from patients with GVHD and without GVHD) formed a higher and bigger round shape and abundant protrusions compared with the total CD4 + CD25- cells (including from patients with GVHD and without GVHD) (Figure 1A,B). The cell surface architecture in the Treg cells showed more irregular microspikes especially in the cell surface center. The irregular microspikes or nano clusters might be the ultrastructure membrane proteins which were reorganized the specific antigens and corresponding to the large change in the structure of the cells surface. Moreover, the CD4 + CD25+ cells with GVHD showed smaller and more irregular shape compared with the CD4+CD25+ cells without GVHD (Figure 1C,D). The cell surface architecture had been seriously deformed, and the cell surface center atrophied and no longer had a regular normal Treg cells' shape.

The biophysical property data of Treg cells from all samples measured by AFM were showed in the Table 1.
The average surface fluctuation Ra of the Treg cells without GVHD were significant increased in comparison with that of the Treg cells with GVHD $(P<0.001)$. However, by measuring the adhesion force of the cells, there was no significant difference in the stiffness between the total CD4 + CD25- cells and the total CD4 + CD25+ cells groups (5.23 $\pm 0.43 v s .5 .51 \pm 0.67, P=0.2807$ ), or between the CD4 + CD25+ cells with GVHD and the CD4 + CD25+ cells without GVHD groups $(5.37 \pm 0.49$ vs. $5.72 \pm 0.31$, $P=0.0710)$.

It is well known that the main complication of alloHSCT is GVHD, an immunological disorder that affects many organ systems, including the gastrointestinal tract, liver, skin, and lungs [4]. Development of novel strategies that use donor leukocyte infusions, non-myeloablative conditioning, and umbilical-cord blood transplantation has helped expand the application of HSCT over the past few years [17]. Moreover, clinical approaches that restore effective immune regulation are therefore an attractive treatment strategy for GVHD. Treg cells which are potent suppressors of immune responses have been a focal point of research studies designed to mitigate the severity of GVHD in both pre-clinical murine models and in early stage clinical trials $[1,3,8]$. In fact, studies in mice have demonstrated that there is a progressive loss of Treg cells during GVHD, and this leads to the emergence of autoreactive proinflammatory donor $\mathrm{T}$ cells. The initial phase of GVHD might be associated with a
A

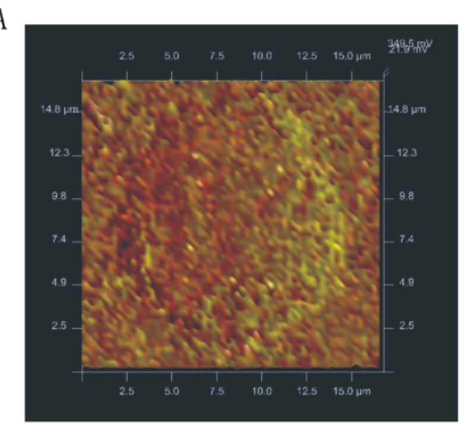

C

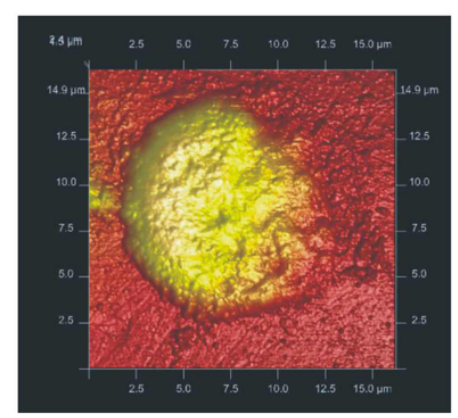

B

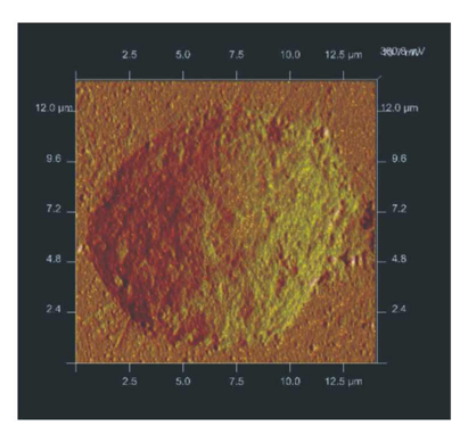

D

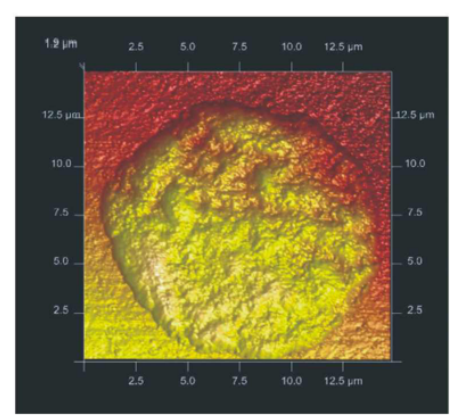

Figure 1 AFM images of Treg cells. A: AFM three-dimensional images of CD4 + CD25+ cells; B: AFM three-dimensional images of CD4 + CD25- cells; C: AFM three-dimensional images of CD4 + CD25+ cells at GVHD onset; D: AFM three-dimensional images of CD4 + CD25+ cells without GVHD. 


\begin{tabular}{|c|c|c|c|}
\hline Groups & $\begin{array}{l}\text { Width }(\mathrm{W}) \\
(\mu \mathrm{m})\end{array}$ & $\begin{array}{l}\text { Peak (H) } \\
(\mathrm{nm})\end{array}$ & $\begin{array}{l}\mathrm{Ra} \\
(\mathrm{nm})\end{array}$ \\
\hline Total CD4 + CD25+ cells & $12.04 \pm 0.24$ & $3.95 \pm 0.27$ & $250.83 \pm 82.39$ \\
\hline Total CD4 + CD25- cells & $10.23 \pm 1.54$ & $3.27 \pm 0.32$ & $110.45 \pm 22.11$ \\
\hline$P$ values* & $<0.001$ & $<0.001$ & $<0.001$ \\
\hline CD4 + CD25+ with GVHD & $11.96 \pm 0.17$ & $3.85 \pm 0.14$ & $191.33 \pm 75.22$ \\
\hline $\mathrm{CD} 4+\mathrm{CD} 25+$ without GVHD & $12.12 \pm 0.13$ & $4.12 \pm 0.21$ & $311.67 \pm 18.45$ \\
\hline$P$ values* & 0.032 & $<0.001$ & $<0.001$ \\
\hline
\end{tabular}

Ra: average roughness $\mathrm{Ra}=(1 / \mathrm{N}) \sum^{N}\left|Z_{n}-\bar{Z}\right| ; \bar{Z}$ : given by the average height within the included areas defined by a region group $\bar{Z}=(1 / N) \sum_{n=1}^{N} Z_{n}$; The $Z$ is the height profile line based on the surface.

*Compared by the Welch test.

decrease of CD4+CD25+ Treg cells in the peripheral blood of patients after allo-HSCT [18]. The CD4+ CD25+ Treg frequencies measured within 24 hrs of GVHD diagnosis were significantly less than patient without GVHD, and correlated inversely with GVHD severity [19]. There will be a promising application in use of adoptive immunotherapy within CD4+ CD25+ Treg cells to treat GVHD patients, however, the biological characteristics of specific Treg cells are still unknown, and that may limit its further application. The AFM applications have the possibility to detect the early subtle changes before significant pathology occur and to give the decision which time to terminate the treatment. Thus, to a certain extent, it is interesting to employ AFM to analysis the biological characteristics of Treg cells after allo-HSCT to further understand the possible mechanisms and role of Treg cells in the treatment and development of GVHD.

In this study, we demonstrated that AFM could be used to simultaneously observe the single membrane proteins and find the details of their surface structures. A common approach to the identification and isolation of Treg cells is the use of CD4+ and CD25+. The Treg cells population represents $2 \sim 10 \%$ of all CD $4+$ cells. Only a subset of CD25+ (the alpha chain of the IL-2 receptor), mostly cells with the highest levels of expression, is considered to be Treg cells [20]. Although the transcription factor forkhead box P3 (FoxP3) is a very specific marker of regulatory $\mathrm{T}$ cells [21], it is an intracellular protein, and the detection of FoxP3 requires permeabilizing the cell membrane. So it cannot be used for sorting the Treg cells without the damage of cell membrane. Thus, we used CD4+CD25+ Treg cells isolation Kit for sorting the Treg cells. The cell surface ultrastructure of CD4 + CD25+ cells at GVHD onset exhibited a regular nanoscale network, and this heterogeneous structure represented a composite of the plasma membrane. And we found that obvious morphological differences occurred in Treg cells without GVHD. The alteration of the cell membrane structure could directly influence the cell function. As one of the advantages of the AFM, it can detect cell membrane proteins and study cell surface structures [11]. By comparing the topography details of the Treg cells surface with GVHD and without GVHD, we found that the multiple irregular microspikes could be observed on the surface of Treg cells in patients without GVHD. The irregular microspikes or nano clusters might be the ultrastructure membrane proteins. These results indicated that such remarkable biological morphology changes in the cell membrane structure of Treg cells might be related to specific activation of Treg cells which could inhibit the development of GVHD. The absence of such the microspikes or nano clusters in Treg cells surface with GVHD might be related to the functional inactivation or dysfunction of Treg cells in GVHD status, and related to the occurrence and development of GVHD.

The mechanical stiffness of individual cells is important in tissue homeostasis, cell growth, division and motility, and the epithelial-mesenchymal transition in the initiation of cancer [22]. In this study, we found that the rigidity of Treg cells from patients with GVHD were similar to Treg cells without GVHD by the detection of adhesion force. That means the biomechanical properties of Treg cells might not be changed significantly on GVHD onset. Even changes in the adhesion force of Treg cells at the beginning of the GVHD were slight, the membrane structure of cell surface had already varied widely, and moreover, the biological morphology changes in the cell membrane structure of Treg cells would certainly cause the physical-mechanical properties changes subsequently with the progress of GVHD. However, further investigation is needed to confirm the physical-mechanical properties changes in Treg cells with GVHD.

\section{Conclusions}

In summary, we compared the morphological feature of Treg cells between patients with GVHD onset and without GVHD by using high resolution AFM imaging mode. The imaging mode allowed us to visualize cell surface properties at nanometer scale as a potential clinical tool for early stage of the GVHD. The research on the changes in cell ultrastructure could help us better understand the structure-function relationships. The study implied the possibility of AFM applications to detect the early subtle changes before significant pathology occur and to give the decision which time to terminate the treatment.

\section{Abbreviations}

Treg: Regulatory T; GVHD: Graft versus host disease; HSCT: Hematopoietic stem cell transplantation; allo-HSCT: Allogeneic hematopoietic stem cell transplantation; AFM: Atomic force microscopy; GVL: Graft-versus-leukemia; CD25: IL-2 receptor a-chain; AML: Acute myeloid leukemia; CML: Chronic myeloid leukemia; PBMCs: Peripheral blood mononuclear cells; Rp-V: Valley-to-peak value; FoxP3: Forkhead box P3. 


\section{Competing interests}

The authors declare that they have no competing interests.

\section{Authors' contributions}

$Y Q L$ and XLW contributed to the concept development and study design. $X W, Z Y J, Y X W$ performed the atomic force microscope measurement, SHC and LJY prepared Treg cells isolation, EKZ was responsible for clinical diagnoses and performed clinical data acquisition. YQL, XLW and XW coordinated the study and helped draft the manuscript. All authors read and approved the final manuscript.

\section{Acknowledgements}

This study was supported by the grants from the National Natural Science Foundation of China (No. 81200388), the Guangdong Natural Science Foundation (No. 10451051501005778, 10451008004004158), the Science and Technology Planning Project of Guangdong Province of China (No. 2012B031800403), the project of the Zhujiang Science \& Technology Star of Guangzhou City (No. 2013027) and Medical Scientific Research Foundation of Guangdong Province (No. B2013183).

\section{Author details}

${ }^{1}$ Key Laboratory for Regenerative Medicine of Ministry of Education, Jinan University, Guangzhou 510632, China. ${ }^{2}$ Institute of Hematology, Jinan University, Guangzhou 510632, China. 3Department of Hematology, First Affiliated Hospital, Jinan University, Guangzhou 510632, China.

Received: 29 January 2014 Accepted: 18 July 2014

Published: 1 August 2014

\section{References}

1. Beres AJ, Drobyski WR: The role of regulatory T cells in the biology of graft versus host disease. Front Immunol 2013, 4:163.

2. Bleakley M, Riddell SR: Molecules and mechanisms of the graft-versusleukaemia effect. Nat Rev Cancer 2004, 4:371-380.

3. Hildebrandt GC, Fazekas T, Lawitschka A, Bertz H, Greinix H, Halter J, Pavletic SZ, Holler E, Wolff D: Diagnosis and treatment of pulmonary chronic GVHD: report from the consensus conference on clinical practice in chronic GVHD. Bone Marrow Transplant 2011, 46:1283-1295.

4. Ferrara JL, Levine JE, Reddy P, Holler E: Graft-versus-host disease. Lancet 2009, 373:1550-1561.

5. X-I W, Liu Q-f: Epstein - Barr virus - associated diseases in allogeneic hematopoietic stem cell transplantation. J Hematol Oncol 2012, 5:A8.

6. Park MJ, Moon SJ, Lee SH, Yang EJ, Min JK, Cho SG, Yang CW, Park SH, Kim HY, Cho ML: Curcumin attenuates acute graft-versus-host disease severity via in vivo regulations on Th1, Th17 and regulatory T cells. PLoS One 2013, 8:e67171.

7. Xuan L, Huang F, Fan Z, Zhou H, Zhang X, Yu G, Zhang Y, Liu C, Sun J, Liu Q: Effects of intensified conditioning on Epstein-Barr virus and cytomegalovirus infections in allogeneic hematopoietic stem cell transplantation for hematological malignancies. J Hematol Oncol 2012, 5:46.

8. Maloy KJ, Powrie F: Regulatory T cells in the control of immune pathology. Nat Immunol 2001, 2:816-822.

9. Lekka M: Atomic force microscopy: a tip for diagnosing cancer. Nat Nanotechnol 2012, 7:691-692.

10. Lekka M, Laidler P: Applicability of AFM in cancer detection. Nat Nanotechnol 2009, 4:72. author reply 72-73.

11. Wang Z, Wu XL, Wang X, Tian HX, Chen ZH, Li YQ: The biophysical property of A549 cells transferred by VEGF-D. Scanning 2014, 36:202-208.

12. Zhang $Y$, Zhang W, Wang S, Wang C, Xie J, Chen X, Xu Y, Mao P: Detection of human erythrocytes influenced by iron deficiency anemia and thalassemia using atomic force microscopy. Micron 2012, 43:1287-1292.

13. Sabattini E, Bacci F, Sagramoso C, Pileri SA: WHO classification of tumours of haematopoietic and lymphoid tissues in 2008: an overview. Pathologica 2010, 102:83-87.

14. Wu X, Zhu K, Du X, Chen S, Yang L, Wu J, Liu Q, Li Y: Frequency analysis of TRBV subfamily sjTRECs to characterize T-cell reconstitution in acute leukemia patients after allogeneic hematopoietic stem cell transplantation. J Hematol Oncol 2011, 4:19.

15. Shi L, Chen S, Lu Y, Wang X, Xu L, Zhang F, Yang L, Wu X, Li B, Li Y: Changes in the MALT1-A20-NF-kappaB expression pattern may be related to T cell dysfunction in AML. Cancer Cell Int 2013, 13:37.
16. da Rosa EL: Kinetic effects of $\mathrm{TiO} 2$ fine particles and nanoparticles aggregates on the nanomechanical properties of human neutrophils assessed by force spectroscopy. BMC Biophys 2013, 6:11.

17. Welniak LA, Blazar BR, Murphy WJ: Immunobiology of allogeneic hematopoietic stem cell transplantation. Annu Rev Immunol 2007, 25:139-170.

18. Schneider M, Munder M, Karakhanova S, Ho AD, Goerner M: The initial phase of graft-versus-host disease is associated with a decrease of CD4 + CD25+ regulatory T cells in the peripheral blood of patients after allogeneic stem cell transplantation. Clin Lab Haematol 2006, 28:382-390.

19. Magenau JM, Qin X, Tawara I, Rogers CE, Kitko C, Schlough M, Bickley D, Braun TM, Jang PS, Lowler KP, Jones DM, Choi SW, Reddy P, Mineishi S, Levine JE, Ferrara $J$, Paczesny S: Frequency of CD4(+)CD25(hi)FOXP3(+) regulatory $T$ cells has diagnostic and prognostic value as a biomarker for acute graft-versus-host-disease. Biol Blood Marrow Transplant 2010, 16:907-914.

20. Seddiki N, Santner-Nanan B, Martinson J, Zaunders J, Sasson S, Landay A, Solomon M, Selby W, Alexander SI, Nanan R, Kelleher A, Fazekas de St Groth B: Expression of interleukin (IL)-2 and IL-7 receptors discriminates between human regulatory and activated T cells. J Exp Med 2006, 203:1693-1700.

21. Pellerin $L$, Jenks JA, Begin P, Bacchetta R, Nadeau KC: Regulatory T cells and their roles in immune dysregulation and allergy. Immunol Res 2014, 58:358-368.

22. Fuhrmann A, Staunton JR, Nandakumar V, Banyai N, Davies PC, Ros R: AFM stiffness nanotomography of normal, metaplastic and dysplastic human esophageal cells. Phys Biol 2011, 8:015007.

doi:10.1186/s13628-014-0007-1

Cite this article as: Wang et al:: AFM detection of biophysical characteristics of specific regulatory $\mathrm{T}$ Cells after allogeneic hematopoietic stem cell transplantation. BMC Biophysics 2014 7:7.

\section{Submit your next manuscript to BioMed Central and take full advantage of:}

- Convenient online submission

- Thorough peer review

- No space constraints or color figure charges

- Immediate publication on acceptance

- Inclusion in PubMed, CAS, Scopus and Google Scholar

- Research which is freely available for redistribution

Submit your manuscript at www.biomedcentral.com/submit
C BioMed Central 